\title{
Generation of 4D Access Corridors from Real-Time Multislice MRI for Guiding Transapical Aortic Valvuloplasties
}

\author{
N.V. Navkar ${ }^{1,2}$, E. Yeniaras ${ }^{2}$, D.J. Shah ${ }^{3}$, N.V. Tsekos ${ }^{2}$, and Z. Deng ${ }^{1}$ \\ 1 Computer Graphics and Interactive Media Lab \\ 2 Medical Robotics Lab, Department of Computer Science, \\ University of Houston, Houston, TX 77004, USA \\ 3 Methodist DeBakey Heart \& Vascular Center, Houston, TX 77030, USA \\ \{nvnavkar, eyeniaras2, ntsekos, zdeng\}@cs.uh.edu, djshah@tmhs.org
}

\begin{abstract}
Real-time image-guided cardiac procedures (manual or robotassisted) are emerging due to potential improvement in patient management and reduction in the overall cost. These minimally invasive procedures require both real-time visualization and guidance for maneuvering an interventional tool safely inside the dynamic environment of a heart. In this work, we propose an approach to generate dynamic $4 \mathrm{D}$ access corridors from the apex to the aortic annulus for performing real-time MRI guided transapical valvuloplasties. Ultrafast MR images (collected every $49.3 \mathrm{~ms}$ ) are processed on-the-fly using projections to extract a conservative dynamic trace in form of a three-dimensional access corridor. Our experimental results show that the reconstructed corridors can be refreshed with a delay of less than $0.5 \mathrm{~ms}$ to reflect the changes inside the left ventricle caused by breathing motion and the heartbeat.
\end{abstract}

Keywords: Cardiac Interventions, Real-Time Image-Guided Interventions, 4D Access Corridors, and Magnetic Resonance Imaging.

\section{Introduction}

The advent of real time image guidance, especially combined with robotic manipulators, may offer new opportunities in interventional medicine. Among the procedures that may benefit from image guidance are intracardiac procedures on the beating heart, such as Transapical Aortic Valve Implantation(TA-AVI) that entail access to the aortic annulus via an apical entrance. Such procedures are usually performed under x-ray fluoroscopy or ultrasound guidance. Threedimensional ultrasound is commonly used due to its real-time volumetric data collection and lack of ionizing radiation, and it can be combined with robotic systems to synchronize the motion of a device and the heart [7. Existing literature in the field of image-guided and/or robot-assisted surgeries is vast and inclusive of highly innovative approaches; herein, we only focus on a few most related efforts and it is by no means a comprehensive literature review. 
In TA-AVI visualization of the Area of Operation (AoO) (i.e., the left ventricle $(\mathrm{LV})$ ) is crucial for manual or robot-assisted maneuvering of the interventional tool, that is, constraining interventions inside the dynamic environment of LV without harming healthy tissue. Recently, real-time magnetic resonance imaging (MRI) has emerged as a promising modality for guiding TA-AVI 24] since it offers certain advantages [1], such as: (a) a wide range of contrast mechanisms, (b) on-the-fly adjustment of the imaging parameters, (c) an inherent coordinate system, (d) tracking of interventional tools, and (e) lack of ionizing radiation. Robot-assisted, MRI-guided TA-AVI have also been successfully demonstrated [49]. For MRI guided intracardiac interventions, several studies have introduced the concept of access corridors, trajectories, and virtual fixtures to reach the targeted anatomy. In addition to imaging speed, another important factor is the extraction and visualization of such access corridors. Ren et al. 8] introduced the concept of dynamic virtual fixtures to assist the operator in minimally invasive procedures in the beating heart. With virtual fixtures, abstract sensory information extracted from preoperative dynamic MRI is overlaid to images to avoid unwanted motion of the interventional tool. Recently, researchers have also reported the generation of dynamic 3D access corridors from preoperative short axis CINE MRI 9] and an efficient algorithm to track the motion of specific anatomical landmarks for guidance [10. However, both the approaches used CINE MRI that is slow (i.e., requiring several heart beats for one set) and thus inappropriate for real-time guidance. Notably, real-time MRI can reach a speed of 30ms per image [6].

As real-time MRI evolves, new methods can be pursued to improve guidance. In this work, we demonstrates by proper image selection and scheduling, a slow imagining modality (i.e. the MR scanner) can be used in real-time for guidance by generating sufficient information of dynamic interventional environment. We propose a novel algorithm for computing dynamic access corridors from obliqueto-each-other, real-time MRI slices and demonstrate its usefulness for a TA-AVI. The introduced three-step method introduces computational tools for:

- Dynamic MRI by collecting a number of oblique-to-each-other slices with ultrafast MRI prescribed to image particular areas of interest,

- Extraction of the moving LV/endocardium by calculating boundary points from signal intensity projections on those slices, and

- Generation of dynamic access corridor in form of 3D dynamic meshes from those boundary points inside the moving LV.

\section{Methodology}

Generation of dynamic access corridors is based on two criteria. First, it should ensure that the interventional tool does not harm the moving endocardial wall and other vital structures including mitral valve leaflets, papillary muscles, and chordae tendinae. Second, it should bring the tool to the targeted anatomy (aortic root). In the proposed approach, generation of the dynamic access corridors entails three steps that run in parallel, as shown in Fig. 1, and are described in the following sections. 


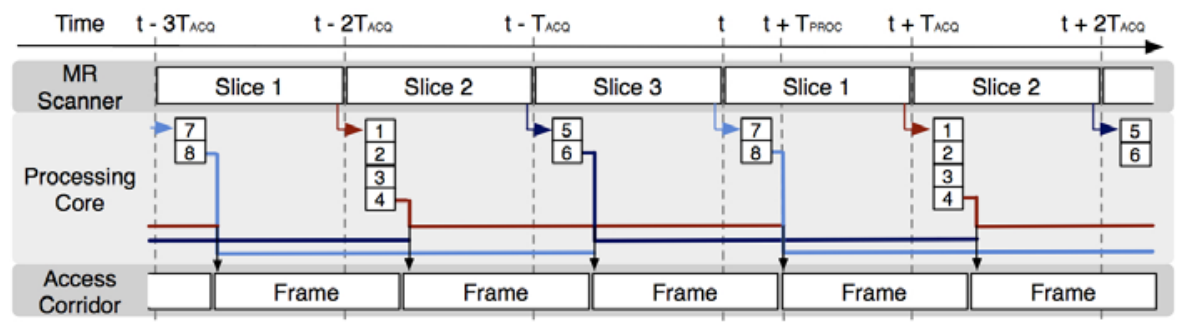

Fig. 1. Illustration of the data collection and processing pipeline: Non-triggered sets of three oblique MR slices are continuously collected (49.3 ms/image) and transferred to the processing core. The core calculates a number of signal intensity projections (4, 2 and 2 for slices 1, 2 and 3 respectively; the numbers 1 to 8 denote the eight signal intensity projections), extracts the boundary points of endocardium and aortic root, organizes and refreshes them (shown by different color lines) to generate a dynamic access corridor. To maintain a uniform frame rate, equivalent to that of MR image collection, data from an individual slice acquisition is retained for the next two acquisition steps until it is refreshed. $T_{P R O C}$ is the time lag between MR collection and refreshing the access corridor.

\subsection{Collection of Dynamic MR Data}

In our particular TA-AVI paradigm, imaging of the AoO is performed by continuously collecting three oblique-to-each-other slices with ultrafast MRI (at $T_{A C Q}=$ $49.3 \mathrm{~ms} /$ slice). These slices were selected preoperatively by the interventionalist physician to image particular areas of interest (shown in Fig. 2a). In particular: (1) Slice $1\left(I_{1}(t)\right)$ is a long axis of view that images the apical region of heart and the aortic and mitral valves, $(2)$ Slice $2\left(I_{2}(t)\right)$ is also a long axis view that depicts the apical region of the heart, and (3) Slice $3\left(I_{3}(t)\right)$ is a short axis view prescribed to include the aortic annulus and the LV.

\subsection{Extraction of Boundary Points}

As each individual MR image is collected, it is sent to a computer via the MR scanner local network, where it is processed to extract the endocardium-LV boundary points $\mathbf{X}_{\mathbf{i}, \mathbf{j}}(t)$ ( $\mathrm{i}=$ slice index, $\mathrm{j}=$ marker index $)$. The boundary points are calculated from signal intensity projections that correspond to bands $\operatorname{Pr} B_{i}$, with a width $W_{i}$ and a length $L_{i}$ (where $\mathrm{i}=1$ to 8 ). Those bands are assigned preoperatively by the operator on scout MR runs, to monitor the motion of specific areas of the endocardium. In this case, the operator selects four projection bands on slice $I_{1}(t)$ and two each on slices $I_{2}(t)$ and $I_{3}(t)$ (shown in Fig. 2 a). The selection of the projection bands is arranged so that $\operatorname{Pr} B_{1}, \operatorname{Pr} B_{2}, \operatorname{Pr} B_{5}$, and $\operatorname{Pr} B_{6}$ depict the motion of the endocardium, whereas $\operatorname{Pr} B_{3}, \operatorname{Pr} B_{4}, \operatorname{Pr} B_{7}$, and $\operatorname{Pr} B_{8}$ capture the motion of aortic root.

To calculate the boundary points in the coordinate system of the MR scanner, the following algorithm is used. For each projection band, a local $2 \mathrm{D}$ coordinate system is defined on the slice with its origin set at the center of the projection 


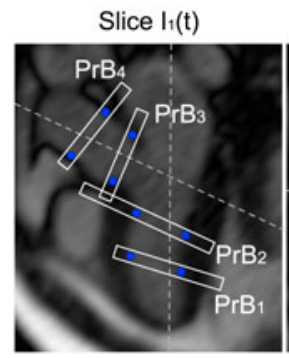

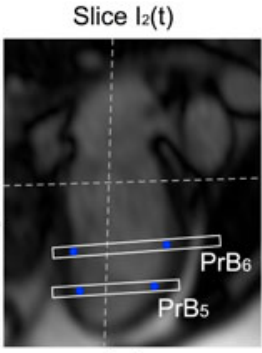

(a)
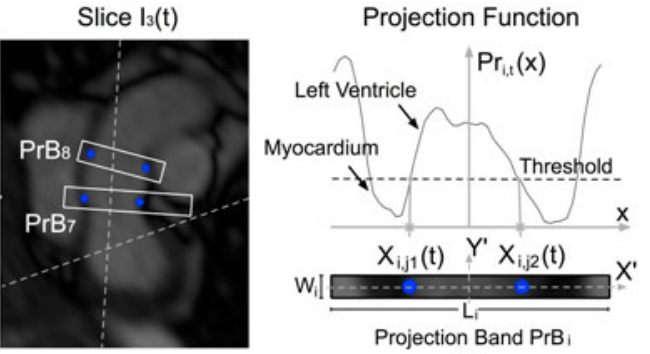

(b)

Fig. 2. (a) Example of the three slices $\left(I_{1}(t), I_{2}(t)\right.$ and $\left.I_{3}(t)\right)$ and the eight operator prescribed projection-bands $\left(\operatorname{Pr} B_{1}\right.$ to $\left.\operatorname{Pr} B_{8}\right)$. The dashed lines on any one of the slices show the intersection of other two slices, and the blue dots correspond to the boundary points. (b) Representative Projection $\operatorname{Pr}_{i, t}(x)$ generated from the projection-band.

band $\operatorname{Pr} B_{i}$ and the axes parallel and orthogonal to its length (Fig. $2 \mathrm{~b}$ ). A projection profile $\operatorname{Pr}_{i, t}(x)$ is generated along the local $\mathrm{X}$-axis of the band. For each value of $x, P r_{i, t}(x)$ represents the averaged signal intensity along its width, i.e. from $\left(x, W_{i} / 2\right)$ to $\left(x,-W_{i} / 2\right)$ at time t.

In the TrueFISP images, the myocardium exhibits lower signal intensity as compared to the LV, and on the projection signal intensity appears as a deep. To identify this deep, a threshold is applied and an algorithm traverses the projection function along the $X$-axis starting from its center and moving along both directions. The threshold is also selected manually from the scout MR scans using a custom GUI gadget. A point is marked on the $X$-axis if the value of $\operatorname{Pr}_{i, t}(x)$ falls below the threshold. After this step, two boundary points $\mathbf{X}_{\mathbf{i}, \mathbf{j} \mathbf{1}}(t)$ and $\mathbf{X}_{\mathbf{i}, \mathbf{j} \mathbf{2}}(t)$ are extracted on the slice $i$ at time $t$ (indexing of the boundary points with respective projection bands is given in Table 1). It takes 9 to 12 minutes for cardiac MR technicians to set up the initial parameters for the pipeline.

\subsection{Generation of 4D Dynamic Access Corridor}

In this step, the dynamic access corridor at time $t$ is represented with a triangular mesh $\mathbf{M}(\mathbf{t})$ and is generated from the boundary points using a two-stage mesh reconstruction process (Fig. $3 \mathrm{a}$ ). In the first stage, a coarse structure is created using the boundary points. The eight boundary points that correspond to the motion of the endocardium, extracted from $\left\{\operatorname{Pr} B_{i}\right\}(\mathrm{i}=1,2,5$ and 6$)$, are interconnected to define a coarse mesh $\tilde{\mathbf{M}}_{\mathbf{E}}(t)$. Similarly, a coarse mesh $\tilde{\mathbf{M}}_{\mathbf{A}}(t)$ is defined for the aortic root from the remaining boundary points, extracted from $\left\{\operatorname{Pr} B_{i}\right\}$ ( $\mathrm{i}=3,4,7$ and 8$)$. The region between the two meshes is created by using Kochanek-Bartels curves [3]. As an example (shown in Fig. 3a) , curve $\mathbf{c}_{\mathbf{1}, \mathbf{t}}(u)$ is defined by the boundary points $\mathbf{X}_{\mathbf{1 , 1}}(t), \mathbf{X}_{\mathbf{1}, \mathbf{3}}(t), \mathbf{X}_{\mathbf{1 , 5}}(t), \mathbf{X}_{\mathbf{1}, \mathbf{7}}(t)$ and is interpolated between the points $\mathbf{X}_{\mathbf{1}, \mathbf{3}}(t)$ and $\mathbf{X}_{\mathbf{1}, \mathbf{5}}(t)$. Curves $\mathbf{c}_{\mathbf{2}, \mathbf{t}}(u), \mathbf{c}_{\mathbf{3}, \mathbf{t}}(u)$, and $\mathbf{c}_{\mathbf{4}, \mathbf{t}}(u)$ are defined in a similar manner. It is noteworthy that the tangential properties of the curve can be altered to adjust the deflection of the access corridor from the endocardium to the aortic root. 


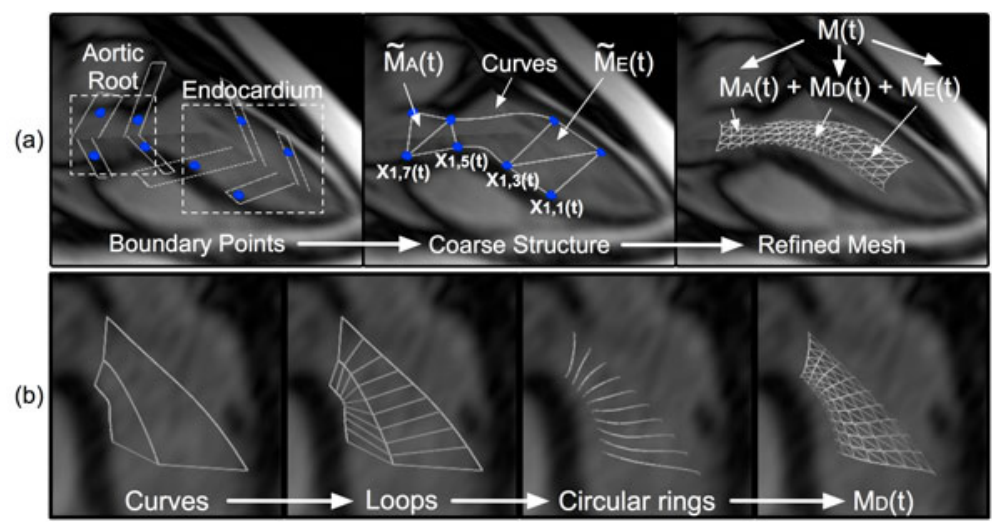

Fig. 3. (a) Generation of dynamic access corridor $\mathbf{M}(\mathbf{t})$ from boundary points (overlaid on slices $I_{1}(t), I_{2}(t)$ and $\left.I_{3}(t)\right)$. (b) Generation of deflection mesh $\mathbf{M}_{\mathbf{D}}(t)$ from the curves (overlaid on slice $I_{1}(t)$ ).

The second stage refines the above coarse mesh structure. Points at regular intervals on the curves $\mathbf{c}_{\mathbf{1}, \mathbf{t}}(u), \mathbf{c}_{\mathbf{2}, \mathbf{t}}(u), \mathbf{c}_{\mathbf{3}, \mathbf{t}}(u)$, and $\mathbf{c}_{\mathbf{4}, \mathbf{t}}(u)$ are interconnected to form intermediate loops, which are further subdivided to get circular rings [5. These circular ring are then interconnected to form a deflection mesh $\mathbf{M}_{\mathbf{D}}(t)$ (Fig. 3b). The coarse meshes $\tilde{\mathbf{M}}_{\mathbf{A}}(t)$ and $\tilde{\mathbf{M}}_{\mathbf{E}}(t)$ are also subdivided to get finer meshes $\mathbf{M}_{\mathbf{A}}(t)$ and $\mathbf{M}_{\mathbf{E}}(t)$, respectively [5. Since for the three meshes $\mathbf{M}_{\mathbf{D}}(t)$, $\mathbf{M}_{\mathbf{A}}(t)$, and $\mathbf{M}_{\mathbf{E}}(t)$ we use the same subdivision scheme (with the same number $(n=2)$ of iterations), the three meshes can be stitched together to form the final mesh $\mathbf{M}(\mathbf{t})$ without altering the positions of the boundary vertices.

Our refinement process is conservative in nature as the mesh $\mathbf{M}(\mathbf{t})$ is confined within the region defined by the coarse meshes $\tilde{\mathbf{M}}_{\mathbf{A}}(t)$ and $\tilde{\mathbf{M}}_{\mathbf{E}}(t)$ and the curves $\mathbf{c}_{\mathbf{i}, \mathbf{t}}(u)$ (where $\mathrm{i}=1$ to 4 ). The region defined by the coarse meshes and the curves is confined by the anatomical structures. For every time frame, the final mesh $\mathbf{M}(\mathbf{t})$ will always have the same number of vertices and faces. Thus, the vertices on the mesh could be indexed based on the nearby anatomical structure. This allows the corridor to provide an adaptive guidance mechanism for an interventional region. The total time required for computing the mesh from the boundary points is given by $T_{M E S H}$.

\section{Experimental Studies and Discussion}

The proposed approach was experimentally tested and validated on a Siemens 1.5T Avanto MR scanner. Multislice non-triggered and free-breathing imaging was performed with a true fast imaging with steady-state precession (TrueFISP), at a $T_{A C Q}=49.3 \mathrm{~ms}$ per slice (Pixel Spacing: $1.25 \times 1.25$; FOV: $275 \times 400$; TR: 49.3ms; TE: 1ms; Matrix: 320x200; and Slice Thickness: 6mm). The computational core was implemented on a dedicated PC (Intel $3.2 \mathrm{GHz}$ processor with 

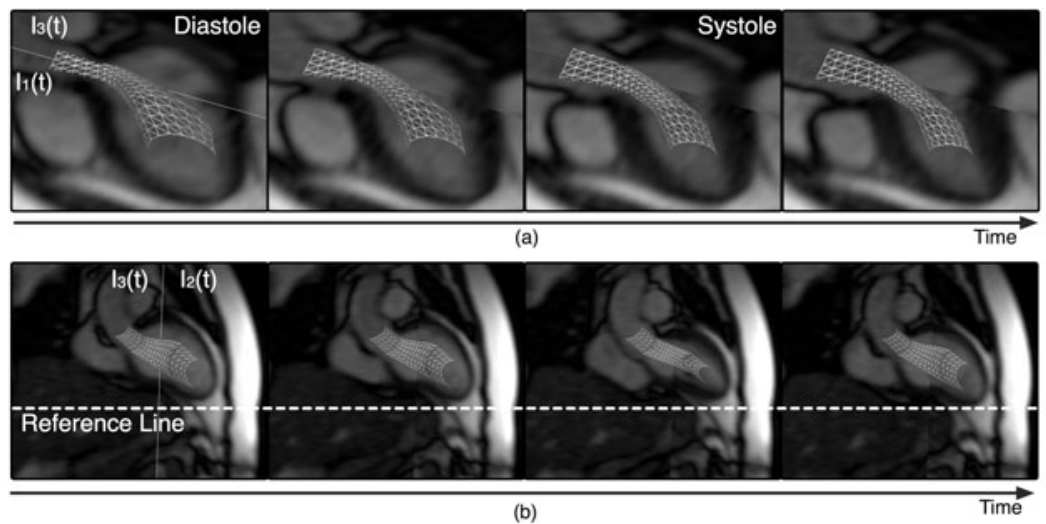

Fig. 4. Four selected frames from the stream generated by the computational core showing the dynamic access corridor overlaid to the real time MR images (a) $I_{1}(t)$, $I_{3}(t)$ and (b) $I_{2}(t)$ and $I_{3}(t)$. In (b) the dashed line is included for appreciation of motion caused by breathing.

9GB RAM). Fig. 4 shows an example outcome of the access corridor superimposed onto two slices (out of three) for the sake of clarity. The deformation of the access corridor secondary to heart beating, as well as its relative motion due to free breathing, can be appreciated in those frames. For all the time frames in our studies, we found the access corridor never collided with the endocardium and aortic root depicted on the real-time MR images. The corridor acts as a 'base mesh' and could be further processed as per the needs of intervention.

With the interleaved multislice MRI, each individual slice is refreshed every $3 T_{A C Q}$. Thus, one interesting question is to what degree this reduced (one third) refreshing rate may result in loss of information. To assess this, we performed experiments that continuously collected only one slice (every $49.3 \mathrm{~ms}$ ) and extracted the boundary points. Variations with time in the distance of the boundary points from the origin $\left\|\mathbf{X}_{\mathbf{i}, \mathbf{j}}(t)\right\|$ (measured in the local coordinate system of the projection band) were calculated. From those data, two signals were generated (shown in Fig. 5a): the original (i.e. complete series) signal and one that sampled the original every $3 T_{A C Q}$. The difference between the two signals was used to calculate the error due to interleaving (shown in Fig. 5b). The mean error $E_{M}$ and the correlation coefficient $\kappa$ of the two signals for a period of 9 seconds is shown in Table 1. For all the boundary points, $E_{M}$ stays below two pixel spacing.

Table 1 summarizes our analysis results reporting the time required to compute each projection band $\left(T_{P r, i}\right)$ and the time lag $\left(T_{P R O C}\right)$ between MR collection and refreshing the access corridor. Specifically, $T_{P R O C}$ is computed as max $\left(\sum_{i=1}^{4} T_{P r, i}, \sum_{i=5}^{6} T_{P r, i}, \sum_{i=7}^{8} T_{P r, i}\right)+T_{M E S H}$. In the experiment ( parameters described in Table 1), the measured $T_{P R O C}$ was $0.261 \mathrm{~ms}$ (i.e., $1 / 184$ of $T_{A C Q}$ ); and $0.194 \mathrm{~ms}\left(T_{M E S H}\right)$ was used for the meshing step. In general, for different configurations $T_{P R O C}$ stayed below $0.5 \mathrm{~ms}$. 
Table 1.

\begin{tabular}{|c|c|c|c|c|c|c|c|}
\hline Slice & Projectio & $L_{i}(\mathrm{~mm}$ & $W_{i}(\mathrm{~mm})$ & $T_{P r, i}(\mathrm{~m}$ & $\begin{array}{l}\text { Points } \\
\end{array}$ & $E_{M}(\mathrm{~mm}$ & $\kappa$ \\
\hline \multirow{7}{*}{$I_{1}(t)$} & \multirow{2}{*}{$\operatorname{Pr} B_{1}$} & \multirow{2}{*}{55.13} & \multirow{2}{*}{6.13} & \multirow{2}{*}{0.018} & $\mathbf{X}_{1, \mathbf{1}}(t)$ & 1.22 & 0.83 \\
\hline & & & & & $\mathbf{X}_{\mathbf{1}, \mathbf{2}}(t)$ & 1.07 & 0.84 \\
\hline & \multirow{2}{*}{$\operatorname{Pr} B_{2}$} & \multirow{2}{*}{70.40} & \multirow{2}{*}{6.09} & \multirow{2}{*}{0.022} & $\mathbf{X}_{1,3}(t)$ & 1.03 & 0.81 \\
\hline & & & & & $\mathbf{X}_{1,4}(t)$ & 1.57 & 0.87 \\
\hline & \multirow{2}{*}{$\operatorname{PrB}_{3}$} & \multirow{2}{*}{47.05} & \multirow{2}{*}{5.93} & \multirow{2}{*}{0.013} & $\mathbf{X}_{1,5}(t)$ & 1.41 & 0.78 \\
\hline & & & & & $\mathbf{X}_{1, \mathbf{6}}(t)$ & 1.89 & 0.64 \\
\hline & $\mathrm{PrB}_{4}$ & 49.69 & 6.02 & 0.014 & \begin{tabular}{|l|l}
$\mathbf{1}, \mathbf{7}(t)$ \\
\end{tabular} & 1.25 & 0.81 \\
\hline \multirow{4}{*}{$I_{2}(t)$} & \multirow{2}{*}{$\operatorname{Pr} B_{5}$} & & \multirow{2}{*}{5.07} & \multirow[b]{2}{*}{0.021} & & & 0.83 \\
\hline & & 62.84 & & & $|\bar{X}, 2(t)|$ & 1.18 & $|0.83|$ \\
\hline & \multirow{2}{*}{$\operatorname{Pr} B_{6}$} & \multirow{2}{*}{82.53} & \multirow{2}{*}{5.07} & \multirow{2}{*}{0.027} & $\mathbf{X}_{\mathbf{2}, \mathbf{3}}(t)$ & 1.79 & 0.83 \\
\hline & & & & & $\mathbf{X}_{2,4}(t)$ & 1.90 & 0.74 \\
\hline \multirow{4}{*}{$I_{3}(t)$} & \multirow{2}{*}{$\operatorname{Pr} B_{7}$} & \multirow{2}{*}{64.96} & \multirow{2}{*}{18.94} & \multirow{2}{*}{0.043} & $\mathbf{X}_{\mathbf{3}, \mathbf{1}}(t)$ & $\overline{0.60}$ & 0.92 \\
\hline & & & & & $\mathbf{X}_{\mathbf{3 , 2},}(t)$ & 0.43 & 0.95 \\
\hline & \multirow{2}{*}{$\operatorname{Pr} B_{8}$} & \multirow{2}{*}{43.45} & \multirow{2}{*}{10.23} & \multirow{2}{*}{0.017} & $\mathbf{X}_{\mathbf{3}, \mathbf{3}}(t)$ & 0.71 & 0.90 \\
\hline & & & & & $\mathbf{X}_{\mathbf{3}, \mathbf{4}}(t)$ & 0.80 & 0.84 \\
\hline
\end{tabular}

Future work can be geared towards optimizing MR collection in two directions. First, while in this work the flow of information is one-way (i.e., from the MR scanner to the processing core), real-time feedback to the MR scanner can be added to adjust the orientation of the imaging planes on-the-fly based on projections. Second, 2D imaging can be substituted with the collection of actual MR projections, as is the case with navigator echoes (with a 90-180 MR pulse sequence to select a column through the tissue and sample the actual projection from the MR signal). This can further speed up data acquisition (since a complete image is not col-

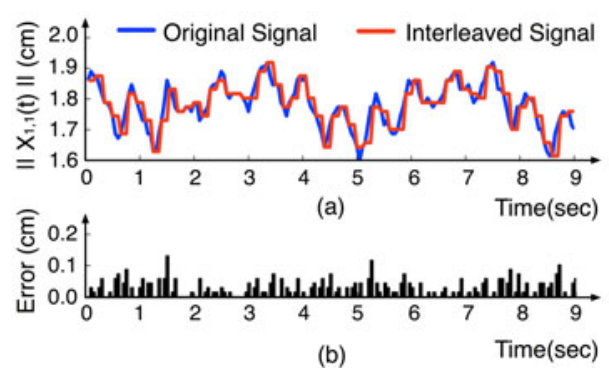

Fig. 5. (a) Comparison of the time-delay effect. The single slice signal is sampled at $20.28 \mathrm{~Hz}$ (i.e. $49.3 \mathrm{~ms} /$ slice) and the multislice at $6.76 \mathrm{~Hz}$ (i.e. $147.9 \mathrm{~ms} / \mathrm{slice}$ ). (b) The error between single and multislice $(n=3)$ acquisition. The "Error" ( $\mathrm{Y}$ axis) is the absolute value of the difference between the two signals. lected) and, in addition, improve the SNR (since a read out projection is collected instead of an image).

\section{Conclusion}

This work proposes an approach to generate $4 \mathrm{D}$ access corridors for performing interventions in the beating heart, from a non-triggered continuously acquired set of oblique-to-each-other MR images. This approach is largely motivated by the challenge associated with the inherent low sensitivity of MRI modality that prevents collecting high SNR, and often high CNR, images in real-time. The reconstructed corridor is virtually refreshed with the same speed as the individual 
MR slices are collected (in this case, $49.3 \mathrm{~ms} /$ image) with a delay of less than $0.50 \mathrm{~ms}$. This dynamic corridor can be used, without or with an additional safety margin (for a more conservative approach), for visual servoing, imagebased robot control, or force-feedback-assisted manual control.

Acknowledgments. This work was supported by the National Science Foundation (NSF) award CPS-0932272. All opinions, findings, conclusions or recommendations expressed in this work are those of the authors and do not necessarily reflect the views of our sponsors.

\section{References}

1. Guttman, M.A., Ozturk, C., Raval, A.N., Raman, V.K., Dick, A.J., DeSilva, R., Karmarkar, P., Lederman, R.J., McVeigh, E.R.: Interventional cardiovascular procedures guided by real-time mr imaging: an interactive interface using multiple slices, adaptive projection modes and live 3d renderings. Journal of Magnetic Resonance Imaging 26(6), 1429-1435 (2007)

2. Horvath, K.A., Mazilu, D., Kocaturk, O., Li, M.: Transapical aortic valve replacement under real-time magnetic resonance imaging guidance: experimental results with balloon-expandable and self-expanding stents. European Journal of Cardiothoracic Surgery 39(6), 822-828 (2010)

3. Kochanek, D., Bartels, R.H.: Interpolating splines with local tension, continuity, and bias control. SIGGRAPH Computer Graphics 18(3), 33-41 (1984)

4. Li, M., Kapoor, A., Mazilu, D., Horvath, K.A.: Pneumatic actuated robotic assistant system for aortic valve replacement under mri guidance. IEEE Transaction on Biomedical Engineering 58(2), 443-451 (2011)

5. Loop, C.: Smooth subdivision surfaces based on triangles (master thesis) department of mathematics, university of utah (1987)

6. McVeigh, E.R., Guttman, M.A., Kellman, P., Raval, A.N., Lederman, R.J.: Realtime, interactive mri for cardiovascular interventions. Academic Radiology 12(9), 1121-1127 (2005)

7. Mebarki, R., Krupa, A., Collewet, C.: Automatic guidance of an ultrasound probe by visual servoing based on B-mode image moments. In: Metaxas, D., Axel, L., Fichtinger, G., Székely, G. (eds.) MICCAI 2008, Part II. LNCS, vol. 5242, pp. 339-346. Springer, Heidelberg (2008)

8. Ren, J., Patel, R.V., McIsaac, K.A., Guiraudon, G., Peters, T.M.: Dynamic 3-d virtual fixtures for minimally invasive beating heart procedures. IEEE Transaction on Medical Imaging 27(8), 1061-1070 (2008)

9. Yeniaras, E., Deng, Z., Syed, M.A., Davies, M., Tsekos, N.V.: Virtual reality system for preoperative planning and simulation of image guided intracardiac surgeries with robotic manipulators. In: Studies in Health Technology and Informatics, vol. 163, pp. 716-722. IOS Press, Amsterdam (2011)

10. Zhou, Y., Yeniaras, E., Tsiamyrtzis, P., Tsekos, N., Pavlidis, I.: Collaborative tracking for MRI-guided robotic intervention on the beating heart. In: Jiang, T., Navab, N., Pluim, J.P.W., Viergever, M.A. (eds.) MICCAI 2010. LNCS, vol. 6363, pp. 351358. Springer, Heidelberg (2010) 